\title{
Tracheoesophageal fistula caused by chemotherapy in primary thyroid lymphoma: a case report
}

\author{
Chun Wang, Zhirong Li, Wuguo Tian \\ Department of Breast and Thyroid Surgery, Daping Hospital, Army Military Medical University, Chongqing, China \\ Correspondence to: Wuguo Tian. Department of Breast and Thyroid Surgery, Daping Hospital, Army Military Medical University, Chongqing 400042, \\ China. Email: tianwuguogood@126.com.
}

\begin{abstract}
Primary thyroid lymphoma (PTL) is a rare malignant tumor of the thyroid, the diagnosis, and treatment of which are still controversial. Although the treatment of thyroid lymphoma causing respiratory obstruction is controversial, chemotherapy is considered the most effective treatment. Tracheoesophageal fistula is an extremely rare complication of chemotherapy. Here, we report a case of respiratory obstruction caused by a thyroid tumor. A 66-year-old female patient visited the hospital with a goiter with dyspnea. The patient was diagnosed with diffuse large B-cell lymphoma of the thyroid by core needle biopsy. After chemotherapy with a cyclophosphamide vincristine prednisone (CVP) regimen, the tumor obviously subsided, but the patient began to experience clinical symptoms such as hoarseness and difficulty drinking. Fiberoptic bronchoscopy and neck computed tomography revealed a tracheoesophageal fistula. The phenomenon of tracheoesophageal fistula resulting from chemotherapy for thyroid lymphoma is extremely rare. Standard treatment for thyroid lymphoma that causes respiratory tract obstruction does not exist. Through clinical case sharing, increase awareness and early recognition are particularly important in order to minimize appropriately the effect of the event and through this case report, so as to provide some suggestions for clinicians. Cases of airway obstruction should be fully evaluated before treatment, and temporary airway protection measures, such as tracheotomy, surgical decompression, and tracheal metal.
\end{abstract}

Keywords: Primary thyroid lymphoma (PTL); tracheoesophageal fistula; chemotherapy; case report

Submitted Mar 18, 2021. Accepted for publication May 10, 2021.

doi: $10.21037 /$ tcr-21-486

View this article at: http://dx.doi.org/10.21037/tcr-21-486

\section{Introduction}

Primary thyroid lymphoma (PTL) is a rare malignant tumor of the thyroid (1). The main clinical manifestations of PTL are painless thyroid enlargement and related compression symptoms, for which rapid and accurate diagnosis is required (2). Sudden enlargement of the thyroid gland compresses the trachea, causing dyspnea (3). The pathogenesis of PTL is still unclear, and it has been confirmed that there is a certain relationship between PTL and Hashimoto thyroiditis (4). Although the treatment of thyroid lymphoma causing respiratory obstruction is controversial, chemotherapy is considered the most effective treatment. PTL that infringes the trachea is rare, and tracheoesophageal fistula caused by chemotherapy is even rarer, with only sporadic cases reported (5-9). Here, we report the case of a patient with respiratory obstruction caused by thyroid lymphoma who developed a tracheoesophageal fistula during chemotherapy. We present the following article in accordance with the CARE reporting checklist (available at http://dx.doi.org/10.21037/ tcr-21-486).

\section{Case presentation}

A 66-year-old Asian female was admitted to the hospital with a 1-year history of a thyroid mass, which had increased in size in the 2 months before her visit. The mass, which was located on the right thyroid gland, had measured approximately $2 \mathrm{~cm} \times 2 \mathrm{~cm}$ when first detected; however, 


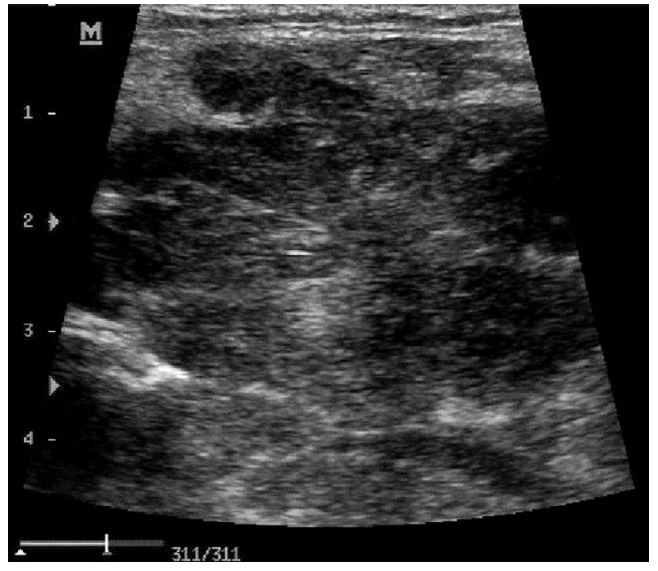

Figure 1 The thyroid gland was diffusely enlarged, the echo of the thyroid was weak, and the internal echo was uneven.

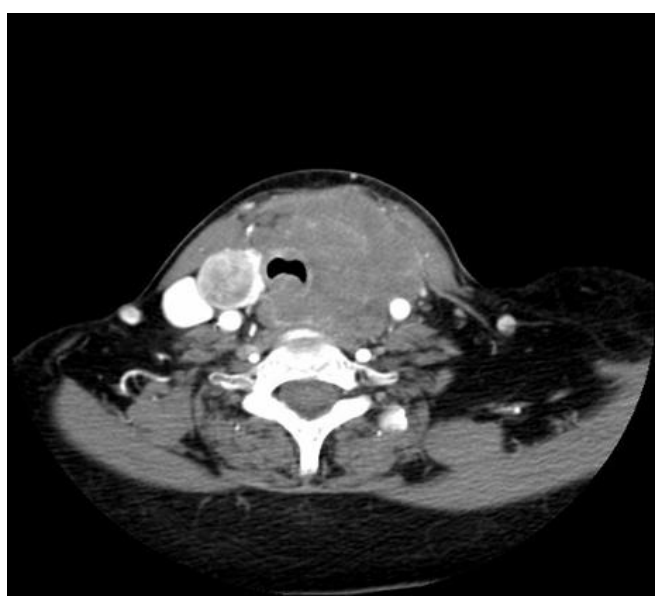

Figure 2 The left thyroid gland was diffusely enlarged with an unclear boundary and closely adhered to the surrounding blood vessels and esophagus. The trachea was compressed and obviously narrowed.

in the 2 months before presentation at our hospital, the patient's neck became swollen, and the mass increased in size to $5 \mathrm{~cm} \times 4 \mathrm{~cm}$. The patient also had difficulty swallowing and throat sounds at night. So far, the patient has not received any treatment. Thyroid ultrasound examination showed that the bilateral thyroid volume was diffuse and enlarged, with an uneven and hypoechoic internal echo and nodular echoes in the thyroid lobe (Figure 1). Neck enhanced computed tomography (CT) revealed bilateral thyroid enlargement and bilateral thyroid mass with a low-density shadow measuring up to $7.6 \mathrm{~cm} \times 4.2 \mathrm{~cm}$, mainly on the left side, which had

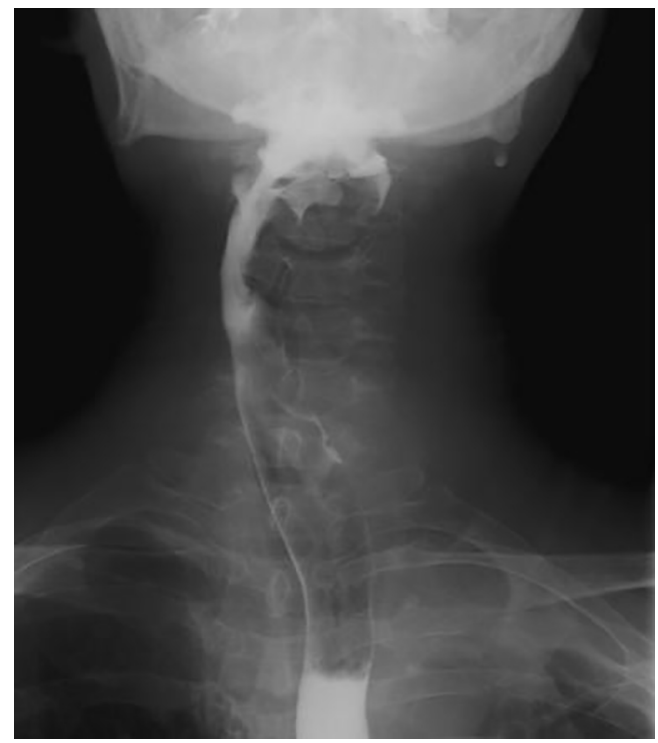

Figure 3 The upper part of the esophagus was compressed, causing stenosis and deformation, the edges were smooth.

uneven density and slight uneven enhancement. Tracheal compression deformation was also observed (Figure 2). Thyroid radioiodine imaging showed that the thyroid had a reduced blood supply and significantly reduced uptake of 99mTco4 on the left side. Esophageal examination found a large arc change in the upper part of the esophagus, measured approximately about $7.2 \mathrm{~cm}$, with local stenosis (Figure 3). Laboratory examination showed: human thyroid-stimulating hormone (hTSH): $0.16 \mathrm{Uiu} / \mathrm{mL}$, thyroxine (T4): $194.6 \mathrm{nmol} / \mathrm{L}$, free T3 (FT3): $7.46 \mathrm{pmol} / \mathrm{L}$, free thyroxine (FRT4): $27.45 \mathrm{pmol} / \mathrm{L}$, thyroglobulin antibody (TG-Ab): 203.59I U/mL, thyroid peroxidase antibody (TPO-Ab): $13.93 \mathrm{IU} / \mathrm{mL}$, and interleukin (IL)6: $42.1 \mathrm{pg} / \mathrm{mL}$. Fine-needle aspiration biopsy showed Hashimoto's thyroiditis morphology in the left mass and atypical changes in some follicular cells. Core needle biopsy revealed a sheet of transparent and transparent lymphoid cell hyperplasia with focal necrosis in the fibrous tissue, together with morphology and immunohistochemistry consistent with diffuse large B-cell lymphoma, non-special type. Immunohistochemistry results: CD10 (+), CD20 (+++), CD21 (+), CD23 (+-), CD3 (scattered+), CD5 (scattered+), CK (individual+), EBER (individual cells+), KI-67 (60\%), LM02 (+), MUM1 (scattered+), Bcl-2 (+), Bcl-6 (+), c-myc (5\%), and p53 (scattered) (Figure 4). Positron emission tomography (PET)/CT revealed left thyroid lymphoma infiltrating the larynx and invading the upper part of the 
trachea, the left glottis, and the left parapharyngeal space, with metastasis to the left cervical area II-IV lymph nodes; the maximum standardized uptake value was 12.81 , and the average value was 9.4. The patient has been diagnosed with PTL accurately.

The patient was prescribed chemotherapy in the form of a cyclophosphamide vincristine prednisone (CVP) regimen, which consisted of: $750 \mathrm{mg} / \mathrm{m}^{2}$ cyclophosphamide, given intravenously, on day $1 ; 1.4 \mathrm{mg} / \mathrm{m}^{2}$ of vincristine, up to a maximal dose of $2 \mathrm{mg}$, given intravenously, on day 1; and $40 \mathrm{mg} / \mathrm{m}^{2}$ of prednisone per day, taken orally, on days 1 to 5 .

Twenty days after completing treatment, despite the

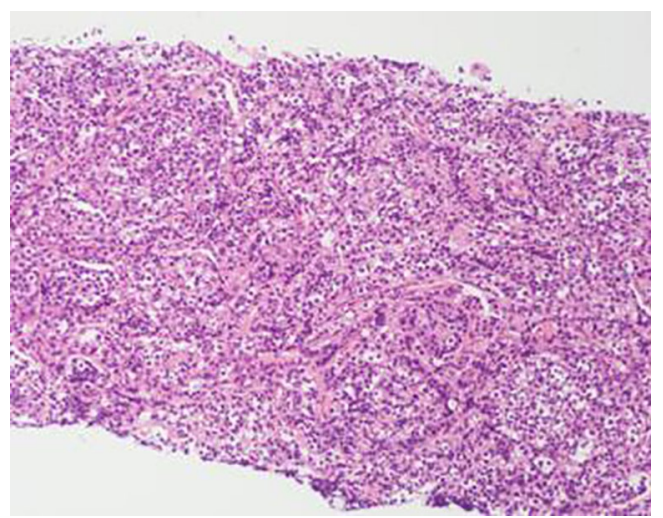

Figure 4 The thyroid follicular structure had disappeared, and there was lymphocytic infiltration and diffuse growth (hematoxylin and eosin, $\times 100$ ). patient's neck mass being significantly reduced in size, her dysphagia and difficulty drinking worsened. CT reexamination of the neck showed that the left neck mass was about $3.2 \mathrm{~cm} \times 4.3 \mathrm{~cm}$ in size, with the lesion surrounding the trachea, left carotid artery, and left vocal cord, as well as the involvement of the retropharyngeal space and visible gas (Figure 5). Bronchoscopy revealed a tracheoesophageal fistula of about $3.5 \mathrm{~cm}$ in length located $0.5 \mathrm{~cm}$ below the glottis, with a large amount of yellow and white necrotic material covering the dense local surface (Figure 6).

The multidisciplinary discussion concluded that after CVP chemotherapy, the thyroid tumor had shrunk significantly, and the tracheoesophageal fistula, as

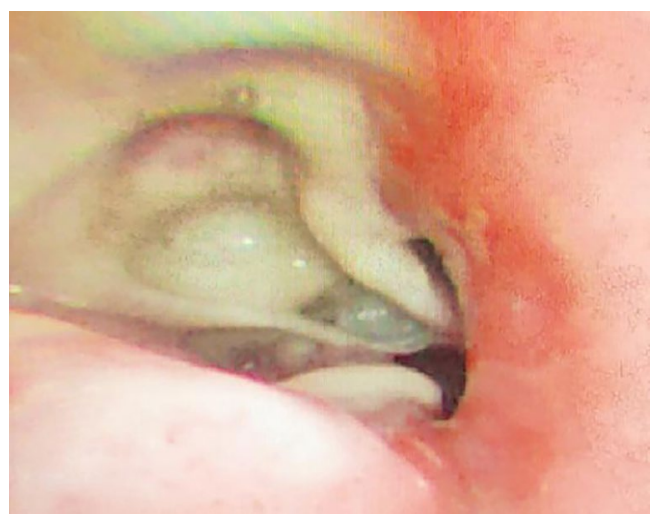

Figure 6 The anterior tracheal wall defect, measuring about $3.5 \mathrm{~cm}$, was seen on bronchoscopy.

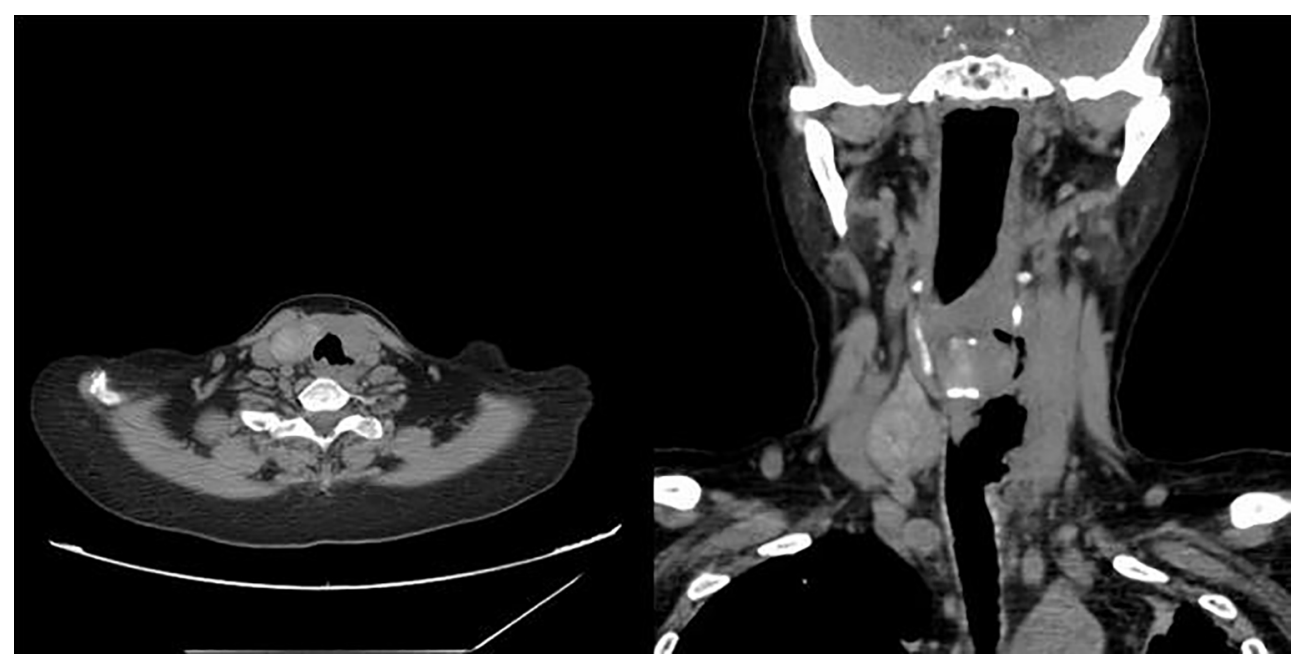

Figure 5 After chemotherapy for thyroid lymphoma, esophageal necrosis and tracheoesophageal fistula occurred. 
determined detected by fiberoptic bronchoscopy, could explain the patient's clinical symptoms of hoarseness and difficulty drinking. At present, the defect of the trachea sidewall combined with cricoid cartilage defect is serious and is challenging to pull and suture. However, to ensure the cross-sectional area of the trachea is sufficient. the sternocleidomastoid muscle clavicular periosteal flap can be used to repair the tracheal defect. After that, the dilator can be placed to repair the esophagus, and a tracheotomy is performed. However, there is no experience in our center, especially the experience of repairing tracheoesophageal fistula caused by chemotherapy for thyroid lymphoma. Unfortunately, after being fully informed of the difficulty of surgery, the patient's family chose to give up her treatment. All procedures performed in studies involving this patient were in accordance with the ethical standards of the institutional and/or national research committee(s) and with the Helsinki Declaration (as revised in 2013). Written informed consent was obtained from the patient's next of kin and family.

\section{Discussion}

Preoperative diagnosis of PTL is difficult, especially in patients with respiratory obstruction; therefore, diagnosing such patients quickly is a challenge for clinicians. Ultrasound is still the preferred imaging method due to its convenience and speed, but it lacks specificity for PTL, which has a very low echo and an unclear boundary. Further, PTL needs to be differentiated from Riedel's thyroiditis and anaplastic thyroid cancer $(10,11)$. Neck enhanced CT is usually atypical. It can be used to measure the tumor size and evaluate the involvement of the trachea, esophagus, and surrounding tissues, including for patients with respiratory obstruction. Compared with CT and MRI, PET-CT has higher diagnostic accuracy in lymphoma, and can be used for staging or therapeutic response evaluation $(12,13)$. In the present case, ultrasound had limited value for the patient, while CT helped to assess the relationship between the thyroid gland and its surrounding tissues. PETCT has obvious value for disease staging and the evaluation of treatment response.

Pathology is the gold standard for the diagnosis of PTL. For patients with respiratory tract obstruction, timely pathological tissue evaluation is the key to diagnosis. Ultrasound-guided fine-needle aspiration cytology (FNAC) is the preferred technique for pathological evaluation of thyroid lesions (14). However, compared with FNAC,
CNB has improved diagnostic accuracy (71\% vs. 93\%) (15). After FNAC, the diagnosis of the patient was not clear. After a full evaluation of its risks, CNB can be used for immunohistochemical diagnosis.

Currently, the treatment of patients with respiratory obstruction is still controversial. Surgical treatment alone cannot improve the prognosis of patients with PTL and is mainly used for patients with mucosa-associated lymphoid tissue (stage IE) confined to the thyroid capsule. However, a tumor exceeding $10 \mathrm{~cm}$ in diameter or a suspected mixed tumor is contraindicated to surgical treatment (16). Patients with severe airway compression may require surgery for symptomatic relief; however, recent evidence has pointed out that high-dose corticosteroids combined with chemoradiotherapy or tracheal stent implantation in a short period can also significantly improve patients' symptoms without surgery (9). There is an increasing amount of evidence to show that combination therapy, such as chemotherapy and local radiotherapy, can improve the overall survival rate of patients. In the case described above, the patient received CVP chemotherapy, and the treatment effect was obvious, with a decrease in tumor size and the relief of dyspnea symptoms; however, 20 days after completing treatment, the patient developed dysphagia and coughing when attempting to drink, and comprehensive consideration found that a tracheoesophageal fistula had occurred.

Chen et al. (9) reported a case of tracheal defect caused by chemotherapy in which no treatment was given for the tracheal wall defect, and the patient's condition was stable. However, the patient had already undergone tracheal intubation before chemotherapy, which damaged the tracheal wall, especially when the tumor compressed the trachea. Although there is some evidence to support that the tracheoesophageal fistula can be repaired, it was reported only in a case report. The mechanism of fistula after chemotherapy is not well understood. In addition to tyrosine kinase inhibitor use, instrumentation of the trachea or esophagus, or a tumor invading vital structures of the neck, including the esophagus and trachea, may be high-risk factors for fistula (17).

In conclusion, thyroid lymphoma is a rare malignant tumor of the thyroid. Tracheoesophageal fistulas caused by chemotherapy are extremely rare. However, clinicians should afford its attention, and especially in patients with thyroid lymphoma with airway obstruction, it is necessary to evaluate the airway before treatment fully. Tracheotomy, surgical decompression, and even tracheal metal stent may 
also be needed, but more studies are needed to confirm the treatment programs of PTL.

\section{Acknowledgments}

Funding: This work was supported by Chongqing Science and Health Joint Medical Project (2020MSXM016).

\section{Footnote}

Reporting Checklist: The authors have completed the CARE reporting checklist. Available at http://dx.doi.org/10.21037/ tcr-21-486

Peer Review File: Available at http://dx.doi.org/10.21037/tcr21-486

Conflicts of Interest: All authors have completed the ICMJE uniform disclosure form (available at http://dx.doi. org/10.21037/tcr-21-486). The authors have no conflicts of interest to declare.

Ethical Statement: The authors are accountable for all aspects of the work in ensuring that questions related to the accuracy or integrity of any part of the work are appropriately investigated and resolved. All procedures performed in studies involving human participants were in accordance with the ethical standards of the institutional and/or national research committee(s) and with the Helsinki Declaration (as revised in 2013). Written informed consent was obtained from the patient's next of kin and family.

Open Access Statement: This is an Open Access article distributed in accordance with the Creative Commons Attribution-NonCommercial-NoDerivs 4.0 International License (CC BY-NC-ND 4.0), which permits the noncommercial replication and distribution of the article with the strict proviso that no changes or edits are made and the original work is properly cited (including links to both the formal publication through the relevant DOI and the license). See: https://creativecommons.org/licenses/by-nc-nd/4.0/.

\section{References}

1. Ha CS, Shadle KM, Medeiros LJ, et al. Localized nonHodgkin lymphoma involving the thyroid gland. Cancer 2001;91:629-35.

2. Green LD, Mack L, Pasieka JL. Anaplastic thyroid cancer and primary thyroid lymphoma: a review of these rare thyroid malignancies. J Surg Oncol 2006;94:725-36.

3. Kumar R, Khosla D, Kumar N, et al. Survival and failure outcomes in primary thyroid lymphoma:a single centre experience of combined modality approach. J Thyroid Res 2013;2013:269034.

4. Ahmed T, Kayani N, Ahmad Z, et al. Non-Hodgkin's thyroid lymphoma associated with Hashimoto's thyrioditis. J Pak Med Assoc 2014;64:342-4.

5. Anagnostis P, Vaitsi K, Tzelepi K, et al. Esophageal perforation in case of thyroid lymphoma. Clin Case Rep 2020;8:1313-4.

6. Hosoya Y, Yokoyama T, Arai W, et al. Tracheoesophageal fistula secondary to chemotherapy for malignant B-cell lymphoma of the thyroid: successful surgical treatment with jejunal interposition and mesenteric patch. Dis Esophagus 2004;17:266-9.

7. Tachibana T, Orita Y, Fujisawa M, et al. Diffuse large B-cell lymphoma of the thyroid which caused esophageal fistula. Esophagus 2015;12:77-81.

8. Melnyk A, Graham NJ, Weber D, et al. Lethal tracheal dissolution during treatment for thyroid lymphoma. Thorax 1995;50:1120-1.

9. Chen C, Tibbetts KM, Tassler AB, et al. Tracheal invasion and perforation from advanced primary thyroid lymphoma: a case report and literature review. Am J Otolaryngol 2013;34:559-62.

10. Gu LS, Cui NY, Wang Y, et al. Comparison of sonographic characteristics of primary thyroid lymphoma and anaplastic thyroid carcinoma. J Thorac Dis 2017;9:4774-84.

11. Zala A, Berhane T, Juhlin CC, et al. Riedel Thyroiditis. J Clin Endocrinol Metab 2020;105:dgaa468.

12. Treglia G, Del Ciello A, Di Franco D. Recurrent lymphoma in the thyroid gland detected by fluorine-18fluorodeoxyglucose PET/CT. Endocrine 2013;43:242-3.

13. Baba S, Abe K, Isoda T, et al. Impact of FDG-PET/ CT in the management of lymphoma. Ann Nucl Med 2011;25:701-16.

14. Tian W, Sun H, He QQ. Expert consensus and operation guide of ultrasound-guided fine needle biopsy of thyroid nodule. Chinese Journal of Practical Surgery 2018;38:241-4.

15. Sharma A, Jasim S, Reading CC, et al. Clinical Presentation and Diagnostic Challenges of Thyroid Lymphoma-A Cohort Study. Thyroid 2016;26:1061-7.

16. Sakorafas GH. What's the role of surgery in the management of primary thyroid lymphoma? Acta Oncol 2011;50:319-20. 
17. Blevins DP, Dadu R, Hu M, et al. Aerodigestive Fistula Formation as a Rare Side Effect of Antiangiogenic Tyrosine Kinase Inhibitor Therapy for Thyroid Cancer.

Cite this article as: Wang C, Li Z, Tian W. Tracheoesophageal fistula caused by chemotherapy in primary thyroid lymphoma: a case report. Transl Cancer Res 2021;10(7):3582-3587. doi: $10.21037 /$ tcr-21-486
Thyroid 2014;24:918-22.

(English Language Editors: J. Reynolds and J. Chapnick) 